\title{
Enhanced Medium Access Control of IP-Based High Speed Wireless LANs
}

\author{
Kuan-Hua Chen, Kwang-Cheng Chen \\ Institute of Communications Engineering, College of Electrical Engineering \\ National Taiwan University, Taipei, Taiwan, 10617, R.O.C. \\ FAX: +886-2-23683824 \\ E-mail: dinose@santos.ee.ntu.edu.tw, chenkc@cc.ee.ntu.edu.tw
}

Abstract - We adapt a general form of multiple access Multi-Layer Collision Anticipation/Resolution (MULCAR) to describe the existing IEEE 802.11 MAC DCF [1]. We further explore the proper way of tailoring the splitting tree to achieve higher throughput and lower delay, primarily for high rate OFDM transmission wireless $\mathrm{LAN}$ at $5 \mathrm{GHz}$.

\section{INTRODUCTION}

Wireless data communication networks have attracted much more interests in recent years, especially worldwide popularity of Internet. After initial application of wireless LANs at the $2.4 \mathrm{GHz}$ ISM band such as IEEE 802.11, Bluetooth and other wireless connection technology are aiming at $2.4 \mathrm{GHz}$ to provide $1-2 \mathrm{Mbps}$ services. With huge investment on NII (national information infrastructure) and GII, wireless access to NII supporting Internet and web data/multimedia traffic is the major technology challenge for wireless networking. Recently, IEEE 802.11 utilized U-NII band at $5 \mathrm{G} \mathrm{Hz}$ to define a new high-speed transmission technology using OFDM (orthogonal frequency division multiplexing) to support up to $54 \mathrm{Mbps}$ services. It is extremely interesting to future wireless applications and as wireless access to NII. However, the IEEE 802.11 medium access control (MAC) remains unchanged and employs CSMA/CA as the fundamental access for distributed coordination function (DCF) and 4-way hand-shaking as the access for point coordination function (PCF). As a matter of fact, due to the nature of IEEE 802.11 MAC, a more efficient MAC compatible with existing IEEE 802.11 CSMA/CA for the applications of high speed wireless data and multimedia networking is very much needed.

Randomly addressed polling has been shown effective for wireless data networks [2] [3]. In later research, there are more interesting results:

- It can be generalized to the concept of MULCAR tree expansion [4]. Therefore, CSMA/CD and CSMA/CA can be shown equivalent to RAP family protocols under the concept of MULCAR.

- IEEE 802.11 4-way hand-shaking to provide better service quality is basically as RAP. More precisely, both follow the same general reliable procedure of wireless access proposed in [2].

This paper was supported in part by the Ministry of Education under the project contract 89-E-FA06-2-4, and in part by the FarEa sTone research grant through national telecommunication program.
The MULCAR was proposed to generalize different multiple access schemes in hope of developing the joint optimal solution under restricted system resource. It describes all the multi-access schemes in two steps, including the Collision Avoidance Tree Expansion (CATE), which emphasize the splitting before data transmission to avoid collisions, and the Collision Resolution Tree Expansion (CRTE) which concerns how to resolve collided nodes. The layer tailoring can be done in the following domains:

- Time domain: e.g. Fixed allocation TDMA.

- Spatial domain: e.g. Direction or distance based spatial domain splitting.

- Feature domain: e.g. Priorities, deterministic traffic type, or stochastical transmitting history.

- Probability domain: e.g. Random back-off as in CSMA family protocols.

- Signaling domain: e.g. Orthogonal signaling as in RAP family protocols.

Random back-off is the most frequently used technique in probability domain splitting. It shares many common characteristics with the orthogonal signaling method while their splitting set uses different system resources. For random back-off splitting, each back-off interval is consumed in a sense of binary carrier sensing. On the other hand, the orthogonal signaling interval can deal with Q-ary carrier sensing. This feature is obtained through the orthogonality in other system resources, such as code or frequency.

Since OFDM in IEEE 802.11 provides 48 sub-carriers for traffic channels, it provides a nature advantage to effectively implement MULCAR (or its simplest form of RAP) with great "generalized carrier sensing" capability to yield high MAC efficiency. We shall take advantage of MULCAR/RAP and OFDM to completely design a hybrid MAC protocol that is much more effective than IEEE 802.11 current MAC as the candidate of high speed wireless LAN MAC. In the mean time, this proposed MAC is compatible with existing MAC so that wireless IP networks such as wireless access to NII, wireless LANs, and wireless Internet, can be much more effectively realized.

\section{SyStem Description}

\section{Assumptions}

1. 48 sub-channel OFDM with each sub-channel QPSK modulation. 
2. Each Sub-carrier tone is orthogonal to each other, thus can be transmitted simultaneously and distinguish-ably received.

3. Non-preemptive service within one polling cycle, that is, newly arrival traffic should wait until the current polling cycle ends.

4. Broadcast of each control signal from the AP (access point) are correctly received by every active node within the cell.

5. The link layer functions such as ARQ are performed at higher layer.

6. Downlink and uplink use the same channel(TDD mode).

\section{Definitions}

1. Frame: not a fixed-length structure, but a functionalspecific one, including the following four messages: acknowledgements, ready, random address, and polling sequence.

2. Polling cycle: One polling cycle contains variable number of frames. It is the total time needed to resolve all the active nodes with non-preemptive assumption.

\section{Frame Format}

There are three types of frames, contention frame, reservation frame, and hybrid frame. The AP in the wireless LAN has the intelligence to decide which frame to be used while performing different functions. Note that the three types of frames share almost the same structure with only slight differences.

1. Contention Frame

The frame structure of a contention frame is in Fig.1, and the AP operating algorithm is as follows:

\begin{tabular}{|l|l|l|l|}
\hline SynciAckID-DIEOB & RA & Polling sequence & U-D \\
\hline
\end{tabular}

Fig. 1. contention frame structure

(a) When the AP is ready to collect the random addresses, it broadcasts a message [EoB] to all mobile nodes under its coverage.

(b) All active mobile nodes simultaneously transmit their own pre-selected random address. Please note that it is done by orthogonal signaling.

(c) The AP listens to all random addresses simultaneously and then decide the polling sequence which is the randomized order of the collected addresses.

(d) All active mobile nodes transmit their up-link data in the assigned slot according to the polling sequence.

(e) If the AP successfully / unsuccessfully receives the packet from any mobile node, it sends a $[\mathrm{PACK}] /[\mathrm{NACK}]$ in the header of the next frame.

(f) Under assumption 3, the polling cycle ends when all packets transmitted within a frame are successfully received by the AP. Otherwise, the polling cycle will repeat step(a)-step(e) again and again until all the transmission intentions are successfully resolved.

2. Reservation Frame

\section{SynclAckID-DIReservation sequence \\ U-D}

Fig. 2. reservation frame structure

Active nodes with time bounded services can inform the AP of the traffic demand and the MS ID in the contention frame. While switched to the reservation frame (as in Fig.2), the AP can do collision-free service by polling the MS ID in the reservation sequence. 3. Hybrid Frame

\begin{tabular}{|l|l|l|l|}
\hline SynclAckID-DIEoB & RA & Polling/Reservation sequence & U-D \\
\hline
\end{tabular}

Fig. 3. hybrid frame structure

It is the combination of the above two types of frames and it simply follows the contention frame operating algorithm. The only difference is the reservation sequence is transmitted along with the polling sequence. The hybrid frame in Fig. 3 has the following two benefits.

(a) It is useful when the AP wants to reduce the frame length variation.

(b) If prompt service is acquired, the AP can immediately serve the node even when the current polling cycle is not ended.

The origin of RAP is to expand the tree expansion domain of collision resolution and collision anticipation. [4] [5] It relies on the orthogonal signaling. Take 802.11[MAC] $\mathrm{DCF}$ for example. The distributed carrier sense nature needs some idle intervals to setup the prioritized message access, the Short Inter-Frame Space (SIFS) and the Distributed Inter-Frame Space (DIFS). Further more, the random back-off after DIFS split the active nodes into more subsets. Although the orthogonal signaling technique is not used here, we can simply find the equivalent tree expansion domain to be like Fig.4. All these idle intervals are waste of channel usage. As a consequence, we adapt centralized carrier sensing in which no idle interval is required. Please note that it can be easily done by the orthogonal multicarrier nature of OFDM. In the following, we will demonstrate the design of our random address detector fitting OFDM physical layer.

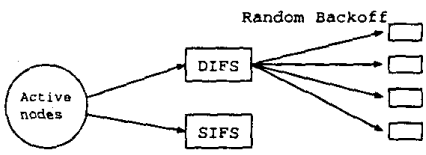

Fig. 4. 802.11 DCF tree splitting

\section{RANDOM ADDREsS TRANSMISSION AND DETECTION}

The key to successful implementation of this enhanced $\mathrm{MAC}$ is the realization of orthogonal signaling and its detection. It turns out that we can take advantage of FFT[6] and OFDM feature by proper signaling to reach this goal 
without any extra hardware or software implementation. Fig.5 and Fig.6 show this concept.

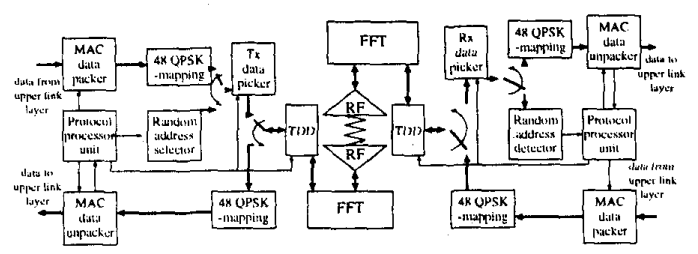

Fig. 5. Adjustments for Random Address $T x$ and $R x$

Consider a system with $i$ active nodes and $N$ subcarriers. For the $b$ th active node in this system, we perform a DFT on the vector $\left\{2 d_{n}\right\}_{n=0}^{N-1}$, where each $d_{n}$ is a complex number of the signal constellation $\left(\alpha_{n}+j \beta_{n}\right)$. The active node can simply select a sub-carrier tone to mark their access intention by selecting one of the following sequences as the $d_{n}$ for the input of the DFT $\{(1,0,0, \ldots, 0),(0,1,0, \ldots, 0) \ldots,(0,0,0, \ldots, 1)\}$. Assume the $m_{b}$ th tone is marked, take the low-pass filtered real part of the DFT result with $f_{m_{b}}=\frac{b}{N \Delta t}$ and $\Delta t=T_{\text {symb }} / N$ :

$$
y_{j}(t)=2 \cos \left(2 \pi f_{m_{b}} t\right)
$$

The AP takes the multiple random addresses by sampling every $\Delta t / 2$ for $2 \mathrm{~N}$ times, the $k$ th sample:

$$
Y_{k}=\sum_{b=0}^{i} y_{b}\left(k \frac{\Delta t}{2}\right)=2 \sum_{b=0}^{i} \cos \left(\frac{2 \pi m_{b} k}{2 N}\right)
$$

By taking DFT of the samples, the AP can split the contents in each sub-carrier.

$$
\begin{aligned}
z_{i} & =\frac{1}{2 N} \sum_{k=0}^{2 N-1} Y_{k} e^{-j\left(\frac{2 \pi k k}{2 N}\right)} \\
& = \begin{cases}\sum_{b=0}^{i} 2 \delta_{0 m_{b}} & l=0 \\
\sum_{b=0}^{i} \delta_{l m_{\mathrm{b}}} & l=1,2, \ldots, N-1 \\
\text { irrelevant } & l>N-1\end{cases}
\end{aligned}
$$

Here we consider two advanced aspects in the design. First, the orthogonal multi-carrier feature is provided by the nature of OFDM. In the conventional random backoff technique, this feature is left unused. Instead, the tree splitting size is enhanced by increasing the number of the back-off interval. This causes the inefficient usage of the channel. On the other hand, it is possible to implement collision avoidance capability in our design, provided that the power of each sub-carrier tones can be an indicator to the number of users who "ring" the tone as their randomly selected address. This capability, addresses as RAP/CA, enables the base station to avoid the collision without sending an actual RTS packet. RTS packet length together with the resulting silence can be much longer than the random address transmission time.

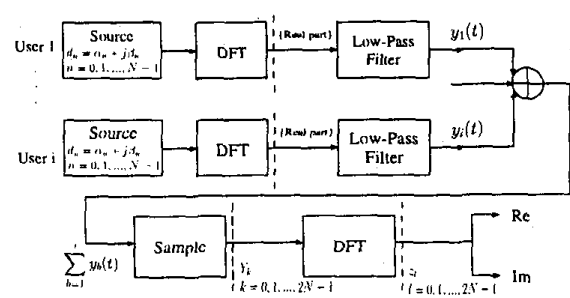

Fig. 6. Functional Diagram of Random Address Tx and Rx

\section{COMPATIBILITY OF CSMA AND RAP FAMILY}

The equivalence between CSMA/CD and RAP protocol has been shown in [7]. Although they are mathematically equivalent, the centralized carrier sensing can avoid hidden terminal problem and is preferred in wireless environment. For the CSMA counterpart, this problem is resolved by the RTS/CTS mechanism in CSMA/CA protocol. The detailed structure is shown in Fig.7.

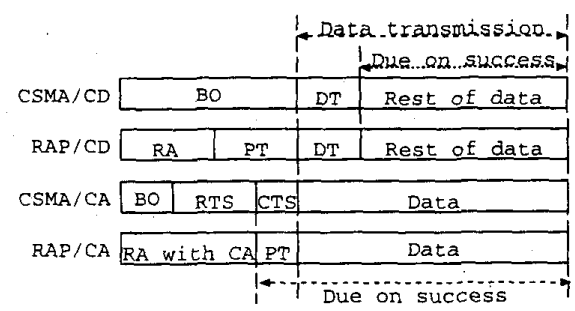

Fig. 7. CSMA vs RAP family

Assume the offered load $G<1$. Let $T_{d}$ be the data transmission time, $T_{i}$ be the idle time in the back-off phase, $T_{R T S}$ be the request-to-send time, $T_{C T S}$ be the clear-tosend time. Define the throughput to be the ratio of successful transmission to the total transmission time. $P_{S u c c}$ is the probability of the first non-empty back-off contains only one user, that is, $\operatorname{Prob}(X=1 \mid X>0)$. Begin with the general form of the one pass throughput performance for CSMA/CA

$$
\eta=\frac{P_{S u c c} \cdot T_{d}}{E\left[T_{i}\right]+T_{R T S}+P_{S u c c} \cdot\left(T_{C T S}+T_{d}\right)}
$$

Comparing with that of RAP/CA:

$$
\eta=\frac{P_{S u c c} \cdot T_{d}}{E\left[T_{R A}\right]+P_{S u c c} \cdot\left(T_{p}+T_{d}\right)}
$$

Please note that the $P_{S u c c}$ in the two schemes are the same by definition. With collision avoidance capability, polling functions as the CTS in CSMA/CA, which implies that $T_{p}$, the polling time, is equivalent to $T_{C T S}$. The remaining term, $E\left[T_{R A}\right]$, which is the expectation of the ran- 
dom address transmission time normalized to a per-poll basis, will be proved equivalent to the CSMA/CA.

For CSMA/CA the expectation of the idle time before the first attempt of transmission is:

$$
E\left[T_{i}\right]=\frac{e^{-G}}{1-e^{-G}} T_{m s}+T_{R T S}=\frac{1}{1-e^{-G}} T_{m s}
$$

We set set $T_{R T S}=T_{m s}$ in (6), and $T_{m s}$ represents the back-off mini-slot time. This is also the result of $E\left[T_{R A}\right]$ for RAP/CA by substituting $T_{m s}$ with $T_{n r}$, the normalized single random address transmission time. From [8], the expected number of random address being marked with total $p$ addresses and $n$ active users $\left(\frac{n}{p} \triangleq G\right)$ is $p[1-(1-$ $\left.\left.\frac{1}{p}\right)^{n}\right]$. We can further explore for infinite user model:

$$
E\left[T_{R A}\right]=\lim _{\frac{p}{n}=G, n \rightarrow \infty} \frac{p T_{n r}}{p\left[1-\left(1-\frac{1}{p}\right)^{n}\right]}=\frac{1}{1-e^{-G}} T_{n r}
$$

It is then clear that for one pass analysis, CSMA/CA and RAP/CA can be equivalent by setting the RTS packet length equal to the random back-off time. Please note that if the entire process is considered, the performance differs due to sampling with replacement (for CSMA) and without (for RAP) as stated in [7].

\section{Grouping improvement anALYSIS}

Define a fixed ratio set to be a contention set with a fixed ratio of the number of users to the size of contention window (or signaling domain). Consider a case in which we can en-group the users before contention to reduce the number of users in a fixed ratio set, we are interested if we can have some performance gain in throughput and delay. In the one pass throughput analysis for RAP in [7]:

$$
\begin{aligned}
S(n, G)= & {\left[G T_{d}\left(1-\frac{G}{n}\right)^{n-1}\right] \cdot\left\{T_{m}+\left(T_{p}+T_{d}\right) G\right.} \\
& \left(1-\frac{G}{n}\right)^{n-1}+\left(T_{p}+T_{c}\right)\left[1-\left(1-\frac{G}{n}\right)^{n}\right. \\
- & \left.\left.G\left(1-\frac{G}{n}\right)^{n-1}\right]\right\}^{-1} \\
\triangleq & \frac{G T_{d} U(n, G)}{D(n, G)}
\end{aligned}
$$

We therefore plot the result of (8) with OFDM physical layer transmission. From the observation of Fig.8, we can conclude that for a given offered load, the throughput is likely to be higher if there are less users in a subset. This can be analytically proved. Take the partial derivative of (8) with respect to $n$ :

$$
\begin{aligned}
\frac{\partial S(n, G)}{\partial n} & =\frac{1}{D^{2}(n, G)} \cdot G T_{d} U(n, G) \cdot\left\{\left(T_{m}+T_{p}+T_{c}\right)\right. \\
& \cdot\left[\frac{n-1}{n} \frac{G}{n-G}+\ln \left(\frac{n-G}{n}\right)\right] \\
& \left.+\left(T_{p}+T_{c}\right) \frac{G}{n^{2}} U(n, G)\right\} \\
& \triangleq \frac{1}{D^{2}(n, G)} \cdot G T_{d} U(n, G) \cdot H(n, G)
\end{aligned}
$$

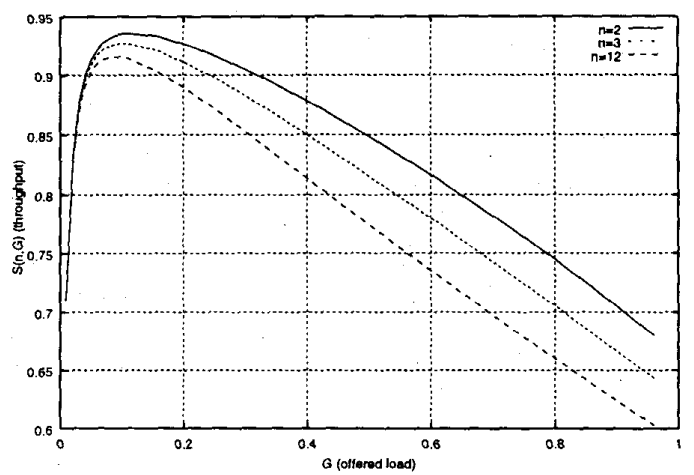

Fig. 8. Throughput vs offered load analysis of RAP with different number of users

Where

$$
\begin{aligned}
U(n, G) & \triangleq \quad\left(1-\frac{G}{n}\right)^{n-1}>0 \\
D(n, G) & \triangleq \quad \text { denumerator of } S(n, G)>0 \\
0<G<1 \quad \text { and } & n \geqslant 2
\end{aligned}
$$

Since $\lim _{G \rightarrow 0_{+}} H(n, G)=0$, we can prove $H(n, G)<0$ by checking its partial derivative with respect to $G$

$$
\begin{aligned}
\frac{\partial H(n, G)}{\partial G} & =\left(T_{m}+T_{p}+T_{c}\right) \frac{G-1}{(n-G)^{2}}+\left(T_{p}+T_{c}\right) \\
& \cdot \frac{1-G}{n^{2}}\left(1-\frac{G}{n}\right)^{n-2} \\
& <\left(T_{p}+T_{c}\right)(1-G)\left[\frac{(n-G)^{n}-n^{n}}{n^{n}(n-G)^{2}}\right] \\
& <0
\end{aligned}
$$

Hence, the derivative in (9) is negative, which means that more users in a fixed ratio set would result in a lower throughput. Although this result is inspiring, some sophisticated control mechanism must be set up to realize it. Recall MULCAR in section I, we can simply adapt other domain to tailor the layers to fit our need. Here, we would like to use the transmission history as additional information in CRTE. Upon each collision, the collided nodes are assigned to form a new set. In each new set, to estimate the number of users in this set, we can simply use the previously collided tone power as an indicator. It is just like to track the operating curve in Fig.8. Once the curve is locked, the BS assigns proper number of random addresses to set the offered load to the optimal point, hence, we can have the optimal throughput for this set.

We can show the stability property of this protocol. With very high probability, there are only two previously collided nodes in a new set. As a result, we can further ap- 
proximate the mean length of the polling cycle for $n$ users.

$$
\begin{aligned}
T_{n} & =n\left\{\frac{T_{m}}{G_{\infty}}+\frac{T_{p}}{G_{\infty}}\left(1-e^{-G_{\infty}}\right)+T_{d} e^{-G_{\infty}}\right. \\
& +\frac{T_{c}}{G_{\infty}}\left(1-e^{-G_{\infty}}-G_{\infty} e^{-G_{\infty}}\right)+\left(1-e^{-G_{\infty}}\right) \\
& \left.\cdot\left[\left(T_{p}+T_{d}\right)+\left(\frac{G_{2}}{2-G_{2}}\right)\left(\frac{T_{p}}{2}+\frac{T_{c}}{2}+\frac{T_{m}}{G_{2}}\right)\right]\right\}
\end{aligned}
$$

where

$$
\begin{aligned}
G_{\infty} & =1-\frac{T_{p}+T_{c}}{T_{m}+T_{p}+T_{c}} e^{-G_{\infty}} \\
G_{2} & =\frac{2 \sqrt{T_{m}}\left(\sqrt{T_{m}+T_{p}+T_{c}}-\sqrt{T_{m}}\right)}{T_{p}+T_{c}}
\end{aligned}
$$

Thus for $\lambda<\frac{n}{T}$, the system is stable. Here we show some example values of $\lambda_{M A X}$ in the following table among which the first row is used as the system parameter for simulation.

\begin{tabular}{|c|c|c|c|c|}
\hline$T_{m}$ & $T_{p}$ & $G_{2}$ & $G_{\infty}$ & $\lambda_{M A X}$ \\
\hline 0.003900 & 0.000977 & 0.117290 & 0.085627 & 0.917652 \\
\hline 0.075470 & 0.018870 & 0.415979 & 0.331983 & 0.697631 \\
\hline 0.010870 & 0.002720 & 0.187680 & 0.139757 & 0.868114 \\
\hline
\end{tabular}

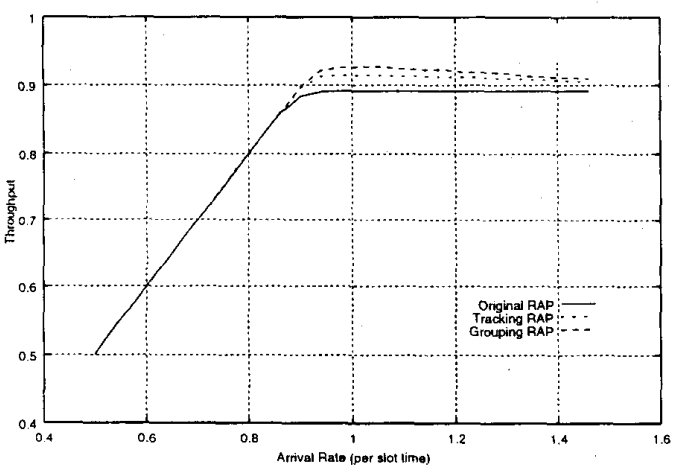

Fig. 9, Throughput

From Fig. 9 and Fig.10, we can observe that the grouping RAP has higher throughput and lower delay than adaptive RAP [7]. However, once we estimate the number of users in a contention set, we can track its optimal operating point on the estimated operating curve as in fig.8. This will undoubtedly lead us to better performance as proved in sectionV.

At the mean time, the necessary grouping information known to the AP turns out to be an important issue. If the AP knows exactly how many users there are in a given set, each set can be processed independently. On the other hand, we know that with high probability that the collisions occur in pairs. However, in a heavy load condition, this property is somewhat weakened. As a result, the curve of grouping RAP bends back to approximate that of adaptive RAP in both throughput and delay.

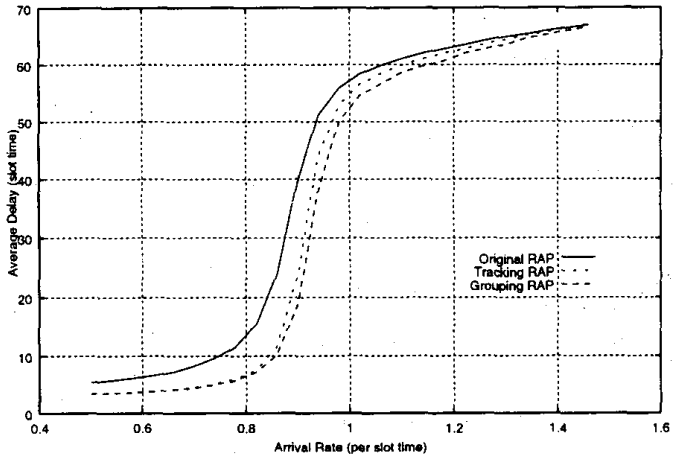

Fig, 10. Delay

\section{Vi. Conclusion}

By taking advantages of the concept for MULCAR, we proposed an enhanced protocol for IEEE 802.11 MAC compatible with the original CSMA/CA scheme. RAP/CA utilizing OFDM physical layer can outperform the CSMA/CA due to the orthogonal multi-carrier nature of OFDM being used as the expansion of random address for RAP. The critical random (multiple) address detector was designed for OFDM transmission with little extra processing to realize the proposed enhanced MAC.

From MULCAR point of view, each layer has its own characteristics. Our future goal is to develop a general algorithm which can collectively consider all the available system resources, properly tailor, and allocate them for multimedia networks, such that the reconfigurable networks [9] can be realized more smoothly and effectively.

\section{REFERENCES}

[1] "Telecommunications and information exchange between systems - Local and Metropolitan networks - Specific requirements - Part 11: Wireless LAN Medium Access Control (MAC) and Physical Layer (PHY) specifications", IEEE Standard for Information Technology, 1999.

[2] K. C. Chen, "Medium access control of wireless LANs for mobile computing," in IEEE Networks, Sep. 1994

[3] K.C. Chen, C. H. Lee, "RAP - a novel medium access control protocol for wireless data networks," in IEEE Global Telecommunications Conference, 1993.

[4] Y. K. Sun, K. C. Chen, D. C. Twu, "Generalized tree multiple access protocols in packet switching networks," in IEEE PIMRC, 1997.

D. Bersekas, R. Gallager, Data Networks, 2nd ed.

6) S. B Weinstein, Paul M. Ebert, "Data' transmission by frequency-division multiplexing using the discrete Fourier transform," in IEEE Transactions on Communication Technology, Oct. 1971 .

[7] C. S. Chang, K. C. Chen, M. Y. You, and J. F. Chang, "Guaranteed quality-of-service wireless access to ATM networks" in IEEE Journal on selected areas in communications, Jan. 1997.

[8] D. C. Twu, K. C. Chen, "Adaptive control strategy for the multilayer collision resolution protocol" in IEEE INFOCOM, 1997.

9] Z J. Haas "On the performance of a medium access control scheme for the reconfigurable wireless networks" in MILCOM g7 Proceedings, 1997. 